\title{
Discours
}

Revue de linguistique, psycholinguistique et

informatique. A journal of linguistics, psycholinguistics and computational linguistics

$21 \mid 2017$

Varia

\section{L'influence des présuppositions sur les témoignages sollicités par questions}

Elizabeth Allyn Smith et Myriam Raymond-Tremblay

\section{OpenEdition \\ Journals}

Édition électronique

URL : http://journals.openedition.org/discours/9401

DOI : 10.4000/discours.9401

ISSN : 1963-1723

Éditeur :

Laboratoire LATTICE, Presses universitaires de Caen

Référence électronique

Elizabeth Allyn Smith et Myriam Raymond-Tremblay, « L'influence des présuppositions sur les témoignages sollicités par questions », Discours [En ligne], 21 | 2017, mis en ligne le 22 décembre 2017, consulté le 05 mai 2019. URL : http://journals.openedition.org/discours/9401 ; DOI : 10.4000/ discours.9401 

Revue de linguistique, psycholinguistique et informatique

\title{
L'influence des présuppositions sur les témoignages sollicités par questions
}

\author{
Elizabeth Allyn Smith \\ Université du Québec à Montréal (UQAM) \\ Myriam Raymond-Tremblay \\ Université du Québec à Montréal (UQAM)
}

Elizabeth Allyn Smith, Myriam Raymond-Tremblay, «L'influence des présuppositions sur les témoignages sollicités par questions», Discours [En ligne], 21 | 2017, mis en ligne le 22 décembre 2017.

URL: http://journals.openedition.org/discours/9401

Titre du numéro: Varia

Coordination: Nicolas Hernandez \& Christian Raymond 



\title{
L'influence des présuppositions sur les témoignages sollicités par questions
}

\author{
Elizabeth Allyn Smith \\ Université du Québec à Montréal (UQAM) \\ Myriam Raymond-Tremblay \\ Université du Québec à Montréal (UQAM)
}

Selon Loftus (1975), présupposer une information nouvelle auprès du témoin d'un événement pourrait influencer les témoignages subséquents de ce témoin. La présente étude tente de reproduire ces résultats dans une autre langue (le français) afin d'en apprendre davantage à propos des caractéristiques linguistiques favorisant ou défavorisant la désinformation. Nos soixante participant(e)s ont regardé une vidéo puis ont répondu à un questionnaire dans lequel était présupposée (a) une information vraie (b) une information fausse ou (c) aucune information. La semaine suivante, tous les participant(e)s ont reçu un second questionnaire qui ciblait l'information critique puis ont passé un test de mémoire de travail. Notre analyse (par régressions logistiques binomiales) est centrée sur la proportion de faux rapports selon la condition. Nos données montrent un effet significatif $(p<0,01)$ d'une présupposition vraie qui réduit le taux de faux rapports, ainsi qu'un effet non significatif $(p=0,09)$ des présuppositions fausses. Nous concluons que des questions posées après un événement peuvent influencer le rapport qu'en fera le témoin, mais que la supposition que cet effet serait universel ne rend pas compte des différences possibles selon la langue et la culture des participant(e)s. Finalement, nous discutons les implications de ces résultats pour les techniques d'interrogatoire actuelles à travers la francophonie qui pourraient menacer l'intégrité des témoignages judiciaires.

Mots clés: présupposition, désinformation, arrière-plan, témoignages, mémoire de travail, linguistique légale, français

Loftus (1975) and subsequent established that presupposing new information during witness questioning can influence subsequent eyewitness reports. We report on a study that attempts to replicate these results for another language (French) in order to better understand some of the factors that facilitate (or not) disinformation. Our sixty participants watched a video of an attempted robbery and answered questions in which the existence of an element was (a) a true presupposition, (b) a false presupposition, or (c) not presupposed. A week later, all participants received a second questionnaire where the critical information was questioned, followed by a working memory task and a demographic survey. Our analysis (by binomial logistic regression) tests the proportion of false responses to the second survey by condition. Our results show that presuppositions of true information significantly decrease the rate of false responses $(p<.01)$ and that false presuppositions increase the rate of false responses, though not significantly $(p=.09)$, as compared to the control condition. We conclude that while questions after an event will have an effect on witness reports, it is not valid to assume that the specific effects will be universal across languages and cultures. Finally, we discuss the implications of these results for various interview techniques used across Francophone nations that could diminish the integrity of judicial testimony.

Keywords: presupposition, misinformation, background, witness testimony, working memory, legal linguistics, French 
Remerciements: Nous remercions nos deux relecteurs anonymes, dont les commentaires pertinents et intelligents ont contribué à la qualité finale de cet article. Nous remercions également les participants de la journée d'étude en linguistique légale à Trois-Rivières, du $85^{\mathrm{e}}$ congrès de l'Association francophone pour le savoir (ACFAS), et des congrès de I'International Association of Forensic Linguistics (IAFL) et de I'International Language and Law Association (ILLA) pour leurs conseils à la suite de présentations préliminaires de ce travail.

\section{Introduction}

Dans les années 1970, Loftus a ouvert la voie à un champ d'études académiques encore très actif aujourd'hui. L'effet de désinformation qu'elle a trouvé dans ses travaux aujourd'hui classiques (Loftus, 1975; Loftus et al., 1978) a piqué l'intérêt de nombreux chercheurs et chercheuses qui l'ont examiné sous plusieurs angles différents. En bref, l'effet de désinformation indique qu'une personne qui reçoit de l'information après avoir vu un événement, lorsqu'elle sera interrogée plus tard, sera plus susceptible de rapporter l'information reçue après l'événement que l'information qu'elle a encodée, ou aurait dû encoder, pendant l'événement.

Malgré le grand nombre d'études publiées en psychologie à ce sujet, il reste beaucoup à faire, particulièrement d'un point de vue linguistique. En effet, bien que la désinformation soit, dans la grande majorité des études, introduite linguistiquement, très peu d'entre elles ont testé le phénomène dans d'autres langues que l'anglais (Jaschinski et Wentura, 2002; Wyler et Oswald, 2016; Zhu et al., 2010). Quand elles le faisaient, elles ne s'y attardaient pas, possiblement par présomption d'une universalité des effets langagiers sur les souvenirs des événements. Pourtant, l'existence potentielle d'un effet culturel ou linguistique sur la résistance à la désinformation semble être un objet de recherche intéressant; Wyler et Oswald (2016) rapportent d'ailleurs des différences qui, selon elles, pourraient être culturelles entre leurs deux expériences menées en Suisse et celle menée en Allemagne, alors même que les trois ont été conduites en allemand. Vornik et al. (2003) trouvent également que des facteurs sociolinguistiques peuvent influencer l'effet de désinformation. Le second aspect du paradigme de désinformation à avoir attiré notre attention est le mécanisme d'introduction de l'information nouvelle. La présupposition est ici largement utilisée (entre autres, Davis et Schiffman, 1985; Dodd et Bradshaw, 1980; Fiedler et al., 1996; Loftus, 1975; Loftus et al., 1978; Sharman et Powell, 2012; Wyler et Oswald, 2016), mais peu étudiée en elle-même. Nous exposerons d'abord une définition théorique de la notion de présupposition, puis effectuerons une revue de la littérature traitant de ce mécanisme utilisé pour créer un effet de désinformation et de celle s'étant penchée sur une corrélation possible avec la mémoire de travail, avant de définir les objectifs de l'étude présente. 


\section{Revue de la littérature}

\subsection{Définition théorique de la présupposition}

La distinction de plusieurs types de significations, parmi lesquels on retrouve la présupposition et l'assertion sous d'autres noms, existe sous diverses formes depuis des siècles (voir Beaver et Geurts, 2011; Karttunen, 2016, pour des survols). Si Ducrot (1968 et 1969) est parmi les premiers linguistes francophones à nommer et à distinguer le posé et le présupposé d'un énoncé, il tranche entre les deux à l'aide d'un test diagnostique inspiré des débats sur la vérité des phrases en philosophie, auxquels participe notamment Russell (1905), mais aussi Frege (1956). L'étude contemporaine de la présupposition en linguistique demeure intrinsèquement liée aux travaux en philosophie du langage de Strawson (1950) et Stalnaker (1974), entre autres. La propriété saillante d'une présupposition qui permet son identification est la conservation de l'information présupposée même lorsque la proposition principale est affirmée, niée, ou même remise en question. Par exemple, les propositions [1a], [1b] et [1c] présupposent toutes [1d] (Ducrot, 1969:33-35).

[1a] Jacques continue à fumer.

[1b] Jacques ne continue pas à fumer.

[1c] Est-ce que Jacques continue à fumer?

[1d] Jacques fumait autrefois.

Une présupposition n'est donc pas affectée par le fait d'être enchâssée dans la portée d'éléments syntaxiques tels que la négation, l'interrogation, et la subordination. De cette façon, elle peut être différenciée du contenu posé: en [1a], par exemple, le contenu posé - «Jacques fume à l'heure actuelle»-est annulé en [1b]. Ce même principe sert à distinguer les présuppositions des sous-entendus, qui ne restent pas non plus constants à travers toutes ces manipulations. Dans le cas de Jacques, les sous-entendus pourraient ainsi varier au niveau de l'intensité du jugement moral véhiculé.

À la même époque que les travaux fondateurs, Stalnaker (1973) a formulé une définition théorique du savoir partagé et des présuppositions qui est basée sur la structure de l'information, notamment la distinction entre une proposition présentée comme étant une nouvelle information et une proposition d'arrière-plan. Sa définition des présuppositions se lit comme suit:

Un locuteur présuppose $P$ à un moment donné dans une conversation seulement dans le cas où il est disposé à agir, dans son comportement linguistique, comme s'il tenait la vérité de $P$ pour acquise, et comme s'il présumait que ses interlocuteurs reconnaîtraient que c'est ce qu'il fait.

(Stalnaker, 1973: 448; nous traduisons de l'anglais) 
Il est important de noter ici, qu'un locuteur ou une locutrice n'a pas besoin de croire la proposition $P$ pour la présupposer, mais seulement d'agir comme si c'était le cas. C'est ainsi que cette personne peut présupposer une information qu'elle sait être fausse. Une proposition qui a été énoncée par le passé fera nécessairement partie du savoir partagé, soit l'ensemble des propositions tenues pour acquises; si elle est présupposée plus tard dans le discours, il s'agira d'une présupposition appropriée («felicitous» en anglais). Si une proposition qui ne fait pas partie du savoir partagé des interlocuteurs est présupposée, elle sera plutôt inappropriée («infelicitous»). L'appropriété («felicity») d’une présupposition et sa vérité sont donc deux choses différentes, ce que l'on peut vérifier à l'aide d'exemples de présuppositions vraies mais inappropriées et de présuppositions fausses mais appropriées. Dans le premier cas, supposons que quelqu'un possède vraiment un lama comme animal domestique, mais qu'il ne l'a jamais mentionné à sa collègue. $\mathrm{Si}$, en arrivant en retard un jour au travail, il énonçait la phrase [2], la présupposition de l'existence de son lama, déclenchée par le possessif défini mon, serait inappropriée malgré sa vérité (et il est facile d'imaginer que sa collègue exprimerait probablement son étonnement et signalerait le non-respect des règles d'usage d'une présupposition en répondant: «Je ne savais pas que vous aviez un lama!»).

[2] Désolé pour le retard, je devais amener mon lama chez le vétérinaire.

Considérons maintenant le deuxième cas: supposons que quelqu'un n'a pas de lama comme animal domestique, mais qu'il a dit à tout le monde qu'il en avait un, y compris son interlocutrice. Le locuteur arrive en retard et prononce la phrase [2]. Cette fois-ci, les présuppositions de l'existence et de la possession du lama sont appropriées même si elles sont fausses, étant donné qu'elles ont été introduites dans le savoir partagé par le passé. Cette distinction entre appropriété et vérité des présuppositions n'est jamais signalée dans les études psychologiques sur le sujet, où la manipulation d'intérêt est toujours la vérité d'un présupposé. En même temps, la littérature en sémantique et pragmatique formelles s'intéresse presque exclusivement à l'autre facteur, c'est-à-dire l'appropriété d'un présupposé. Nous reviendrons sur cette réflexion dans notre discussion à la fin de l'article.

Il existe plusieurs structures ou mots qui sont reconnus pour être des déclencheurs de présuppositions. Parmi eux se trouvent les syntagmes nominaux définis largement utilisés dans la recherche sur l'effet de désinformation, du type «le chat», «mon chat» ou «Minette». On recense également les déclencheurs suivants: les structures clivées ( ¿C’est le chat qui a mangé mon poulet»), les interrogations en $Q u$ - («Qui a mangé mon poulet?»), les quantificateurs («Certains de mes chats aiment le poulet»), les propositions temporelles («Avant qu'il mange mon poulet, le chat était affamé»), les verbes factifs ( $\mathrm{J}$ e regrette que le chat ait mangé mon poulet») et les verbes de transition («Le chat a continué de manger mon poulet»), entre autres (Van der Sandt, 2015). Ainsi, presque tous les énoncés contiennent au moins une présupposition, ce qui nous aide à construire et à échanger des informations et des histoires sur les bases établies. L'omniprésence et la subtilité des présuppositions augmentent encore l'importance qu'il y a à comprendre les effets cognitifs qu'elles pourraient engendrer. 
Depuis les travaux de Stalnaker, plusieurs auteur(e)s ont avancé des théories importantes sur la présupposition, y compris Karttunen (1974), Beaver (2001) et Fintel (2008). Fintel résume la présupposition ainsi : c'est le fait d'agir comme si la proposition était déjà dans le savoir partagé, ce dernier étant défini pour lui comme l'ensemble des propositions qui ont été acceptées par les interlocuteurs ou interlocutrices, qui sont tenues pour vraies et qui ne sont plus sujettes à discussion, suivant la tradition stalnakerienne. Ses travaux concernent les cas, tels que mentionnés plus haut, où une information présupposée dans un énoncé ne sera pas déjà dans le savoir partagé. Malgré la non-appropriété de la présupposition, lorsque certaines conditions sont réunies, les interlocuteurs ou interlocutrices accommoderont cette information, c'est-à-dire l'accepteront et l'ajouteront au savoir partagé sans devoir interrompre la conversation pour porter l'attention dessus. C'est grâce au procédé d'accommodation qu'il est acceptable de dire, à un groupe d'étrangers qui n'ont aucun moyen de savoir que la locutrice a une sœur:

[3] Je suis désolée de mon retard, j’ai dû aller reconduire ma sœur.

Il est intéressant de noter que nous avons essentiellement la même phrase qu'avant, mais que l'accommodation de l'existence d'une sœur se fait plus facilement que l'accommodation de celle d'un lama. Il apparaît alors que le degré auquel une proposition est inattendue déterminera en partie si elle peut être accommodée sans interruption de la conversation. Le procédé d'accommodation de présuppositions ainsi que le facteur modérateur qu'est l'imprévisibilité nous permettent d'inclure des présuppositions dans un questionnaire sans que cela paraisse anormal aux yeux d'un(e) participant(e). En effet, il est tout à fait naturel de présumer que la personne qui établit un questionnaire connait bien le contenu qui y est interrogé; un(e) participant(e) n'a donc aucune raison de ne pas accommoder une présupposition glissée dans les questions, à moins de clairement se souvenir du contraire ou de disposer d'un indice, contextuel ou autre, qui fournisse une raison de ne pas l'accommoder. Dodd et Bradshaw (1980) montrent que c'est le cas en manipulant la source des questionnaires: lorsque les participant(e)s reçoivent des questionnaires construits par un avocat défendant un criminel, ils/elles sont moins sensibles à l'effet de désinformation. Les participant(e)s ne font pas confiance à l'avocat comme ils/elles font confiance au chercheur anonyme.

Ce que nous venons de décrire constitue une des critiques que McCloskey et Zaragoza (1985) adressent aux travaux de Loftus. Selon eux, les conclusions que cette dernière tire sont trop hâtives, car les participant(e)s pourraient très bien se souvenir qu'ils/elles ont vu autre chose que ce qui est présupposé, mais supposer que la chercheuse qui a monté le questionnaire connaissait mieux l'événement qu'eux/elles et donc se fier à elle plutôt qu'à leurs souvenirs. Les réponses qu'ils/elles indiqueraient dans le questionnaire ne refléteraient ainsi pas nécessairement leurs souvenirs. Or, d'un point de vue très concret et dans une perspective d'application de la recherche au contexte judiciaire, la raison qui pousse un témoin à produire un faux rapport n'est pas pertinente, au contraire du fait qu'une information fausse a 
été communiquée à l'enquêteur ou au tribunal. Pour cette raison et parce que nous pensons que le témoin vis-à-vis de l'enquêteur pourrait avoir la même réflexion que le/la participant(e) à une expérience vis-à-vis du chercheur, nous n'avons pas tenu compte de cette critique dans l'élaboration des questionnaires.

\subsection{L'effet des présuppositions dans des témoignages}

Dans l'expérience de Loftus et al. (1978), les participant(e)s regardent d'abord des diapositives d'un événement au sujet duquel ils/elles pourraient être appelé(e)s à témoigner puis sont exposé(e)s à de l'information supplémentaire par le biais de questions auxquelles ils/elles doivent répondre. Une semaine plus tard, ils/elles reviennent et répondent à de nouvelles questions qui les obligent à choisir entre l'information supplémentaire et celle contenue dans les diapositives. Ces chercheurs sont les premiers à utiliser systématiquement des présuppositions déclenchées par un déterminant défini dans leur paradigme de désinformation. Ils manipulent également la vérité des présuppositions: pour la moitié des participant(e)s, la phrase en [4] contient une présupposition vraie, soit le fait qu'il y avait un panneau d'arrêt/panneau stop (stop sign); pour l'autre moitié, la question présuppose faussement la présence d'un cédez-le-passage (yield sign).

[4] Did another car pass the red Datsun while it was stopped at the stop/yield sign?

Le groupe exposé aux présuppositions vraies obtient des résultats significativement meilleurs que le niveau attendu de participants répondant au hasard, soit $50 \%$; inversement, le nombre de bonnes réponses du groupe exposé aux présuppositions fausses est significativement en deçà de ce même palier. L'expérience 2 utilise le même paradigme, mais introduit un groupe contrôle, qui reçoit la question en [5] où ni le panneau d'arrêt/panneau stop ni le cédez-le-passage ne sont présupposés:

[5] Did another car pass the red Datsun while it was stopped at the intersection?

Dans l'ordre, les groupes qui répondent le mieux sont le groupe ayant reçu une présupposition vraie, le groupe contrôle, puis vient le groupe ayant reçu une présupposition fausse. Cependant, il n'y avait pas assez de participant(e)s pour conduire une analyse statistique sur la significativité de ces résultats. Il s'agit d'une des raisons motivant notre étude: si nous souhaitons éventuellement comparer les effets d'autres manipulations linguistiques à un effet de désinformation «de base», il importe d'abord de vérifier que ces effets de base sont bel et bien significatifs.

Par la suite, ainsi que nous l'avons indiqué plus haut, plusieurs auteur(e)s vont utiliser les présuppositions dans leurs recherches sur l'effet de désinformation, mais peu se penchent spécifiquement sur cette question. Fiedler et al. (1996) confirment que des objets absents de la vidéo originale subséquemment présupposés, comme le panier en [6], sont, lors d'un test de reconnaissance, choisis (à tort) plus souvent que les objets absents mentionnés sans être présupposés, comme en [7].

[6] Was the shopping basket near the wardrobe made of bast? 
Was there a shopping basket standing in the corridor?

La présupposition semble donc être plus puissante pour produire un effet de désinformation que l'assertion, soit le fait de poser directement l'existence d'un objet sans la présupposer.

Sharman et Powell (2012), quant à elles, examinent l'influence du type de questions sur les fausses déclarations révélées par le test de reconnaissance subséquent. Elles trouvent que les questions fermées qui incluent plus de détails (exemple [8]) et que les questions à développement incluant des présuppositions (exemple [9]) sont plus puissantes en termes de suggestivité que les questions fermées non détaillées (exemple [10]). Elles analysent également un autre type de questions qu'elles appellent «closed presumptive questions» (exemple [11]), qui sont biaisées, mais ne contiennent pas de présupposition au sens strictement linguistique du terme.

[8] Did the robber hold up the bank with a shotgun that had a black barrel and a dark brown stock?

[9] Tell me about the shotgun that the robber used to hold up the bank?

[10] Did the robber hold up the bank with a shotgun?

[11] The robber held up the bank with a shotgun, didn't he?

Il est difficile d'étudier à partir de ces données l'interaction entre la présupposition et le type de question étant donné qu'il n'y a pas de question ouverte sans présupposition ni de question fermée avec présupposition linguistique venant de la forme de la question.

En somme, le nombre de travaux portant précisément sur l'action des présuppositions dans l'effet de désinformation est insuffisant pour confirmer les conclusions des chercheurs et chercheuses. De plus, les présuppositions utilisées manquent de variété: toutes les études que nous avons consultées qui y avaient recours (Davis et Schiffman, 1985 ; Dodd et Bradshaw, 1980; Fiedler et al., 1996; Loftus, 1975 et 1977; Loftus et al., 1978; Sharman et Powell, 2012) employaient le même type de déclencheur de présupposition, c'est-à-dire des syntagmes nominaux définis qui présupposent l'existence. Il est ainsi impossible de savoir si le type de présupposition a une influence sur l'effet de désinformation, ni même si les autres types de présupposition auront seulement une influence sur l'effet de désinformation. Toutefois, il faut d'abord répliquer certains de ces effets de base pour vérifier qu'ils sont (a) statistiquement significatifs et (b) présents chez les francophones. Nous testerons donc également les présuppositions d'existence liées aux syntagmes nominaux définis afin de pouvoir, par la suite, tester une variété plus grande de types de présuppositions. Avant de songer à répondre à nos objectifs, il nous faut définir un dernier concept et le relier à l'effet de désinformation: la mémoire de travail. 


\subsection{La mémoire de travail}

Quelques études ont déjà exploré le lien entre les caractéristiques cognitives individuelles et la suggestibilité de personnes à l'effet de désinformation. La personnalité (Liebman et al., 2002), l'intelligence, la perception et la mémoire (Zhu et al., 2010) ont, entre autres, été étudiées sous cet angle.

La capacité de mémoire de travail est également l'une de ces caractéristiques cognitives. Il s'agit d'un système psychologique qui peut stocker et manipuler une quantité d'informations limitée, formant ainsi une interface entre la mémoire à long terme, la perception et l'action (Baddeley, 2003). Un test typique mesurant la mémoire de travail force le/la participant(e) à non seulement garder en mémoire, mais en plus à manipuler plusieurs informations à la fois tout en restreignant l'accès à la mémoire à long terme. Il existe plusieurs types de tests: les tests $n$-back, où le/la participant(e) doit décider si le stimulus est identique à celui présenté $n$ étapes plus tôt; les tests d'empan (span task), où le/la participant(e) doit exécuter une tâche de jugement tout en mémorisant un élément puis, après un nombre croissant de tâches, rappeler tous les éléments mémorisés; les tests de mise à jour de la mémoire (memory updating task), où le/la participant(e) doit mémoriser une série d'éléments puis, à chaque étape, appliquer à l'un de ces éléments une opération mathématique ou de déplacement, par exemple, et enfin donner les résultats de la série entière; et finalement, les tests de tri d'empan (sorting span task), où le/la participant(e) doit juger si un élément est bien ordonné dans la liste de tous les éléments déjà présentés (Schmiedek et al., 2014).

En termes simples, la mémoire de travail est donc le module psychologique qui permet de jongler avec plusieurs informations à la fois. Dans le cas qui nous importe, il est possible que le témoin d'un événement avec une meilleure mémoire de travail ait les ressources nécessaires pour, tout en regardant l'action qui se déroule devant lui, se créer une représentation mentale plus cohérente et complète que celle d'un témoin à la mémoire de travail moins développée (Jaschinski et Wentura, 2002). Nous souscrivons donc à l'hypothèse de Jaschinski et Wentura selon laquelle les participant(e)s possédant une meilleure mémoire de travail seront moins sensibles à la désinformation que les autres, dont les représentations mentales seraient lacunaires et fragmentées.

Cette corrélation entre mémoire de travail et susceptibilité à la désinformation a déjà été relevée. Parmi les travaux les plus exhaustifs, l'étude de Zhu et al. (2010) évalue l'influence de l'intelligence (telle que mesurée par les matrices progressives de Raven [ Raven's Advanced Progressive Matrices»] et la version chinoise de l'échelle d'intelligence pour adultes selon Wechsler [ "Wechsler Adult Intelligence Scale-Revised»], qui testent respectivement les raisonnements abstraits et les connaissances générales, la capacité d'attention, les raisonnements abstraits verbaux, la perception visuelle, la coordination et la perception spatiale), de la perception visuelle et auditive, de la reconnaissance des visages et de la mémoire de travail sur la suggestibilité des participant(e)s à l'effet de désinformation. Le test de mémoire de travail est un test 2-back utilisant des caractères chinois et des lettres tibétaines. Les résultats obtenus par ces chercheurs suggèrent que chacune des mesures est corrélée à la suggestibilité. Les participant(e)s 
plus intelligent(e)s, capables d'une meilleure discrimination perceptuelle, doté(e)s d'une meilleure mémoire de travail et d'une meilleure reconnaissance faciale étaient moins susceptibles de rapporter de fausses informations.

De leur côté, Jaschinski et Wentura (2002) ont mesuré la mémoire de travail par un test d'empan de mots et d'opérations mathématiques (ces dernières agissant comme distracteurs) et sont arrivés à la même conclusion.

Cependant, aucune des deux études n'incluait de présuppositions ou n'instillait sa désinformation au travers de questions; elles utilisaient plutôt des textes. Nous cherchons donc à vérifier que la corrélation sera toujours présente dans ces conditions. Par ailleurs, nous avons choisi une autre mesure de la mémoire de travail, soit l'empan de lecture (Desmette et al., 1995), de façon à vérifier que ce n'est pas le test mais bien la mémoire de travail elle-même qui est corrélée avec la suggestibilité.

\subsection{Objectifs de la présente étude en lien avec les études antérieures}

La présente étude s'intéresse à l'effet de désinformation dans sa dimension concrète et linguistique plutôt que psychologique, c'est-à-dire aux facteurs l'influençant et aux moyens de le réduire bien plus qu'aux mécanismes cognitifs qui en sont la cause (pour des revues sur ces derniers, voir Chrobak et Zaragoza, 2013; Zaragoza et al., 2007). Nous avons donc deux grands objectifs: d'abord, nous souhaitons tester la présence d'un effet de désinformation en français, ce qui n'a, à notre connaissance, jamais été accompli. Si l'effet existe effectivement, comme en allemand ou en chinois (Wyler et Oswald, 2016; Zhu et al., 2010), il nous sera alors possible de le comparer grossièrement avec celui qu'on retrouve en anglais. Le second grand objectif consiste à reproduire l'expérience de Loftus et al. (1978) avec une meilleure puissance statistique de façon à vérifier s'il y a effectivement une différence significative entre les groupes. En d'autres termes, nous nous attendons à ce que le groupe recevant des présuppositions vraies fasse moins d'erreurs dans le test de reconnaissance que le groupe ne recevant pas l'information critique présupposée, qui devrait à son tour mieux réussir que le groupe recevant des présuppositions fausses. Nous comptons également examiner la tendance qui s'est manifestée dans cette même étude selon laquelle il semble que les présuppositions vraies réduisent plus le taux de faux rapports que les présuppositions fausses ne l'augmentent. Enfin, le troisième objectif est mineur et vise à vérifier l'existence d'une corrélation entre capacité de mémoire de travail et réceptivité à l'effet de désinformation.

\section{Méthodologie}

Nous avons repris le paradigme en trois étapes élaboré par Loftus (1975) et Loftus et al. (1978). Les participant(e)s visionnaient d'abord une vidéo d'un accident de voiture. Ils/elles ont ensuite répondu à un premier questionnaire où, par le biais des interrogations, ils/elles étaient exposé(e)s, selon leur condition, à de l'information concordant avec la vidéo, de l'information discordante ou pas d'information supplémentaire du 
tout. Enfin, après un délai d'une semaine, les participant(e)s répondaient à un test de reconnaissance (recognition test) pour que nous évaluions, à travers leurs réponses, l'effet que la désinformation avait pu produire.

\subsection{Participants}

Soixante personnes ( 46 femmes, 14 hommes) ayant entre 18 et 37 ans ont participé à l'étude. Elles ont été recrutées selon deux méthodes: des courriels ont été transmis à la population étudiante via les secrétariats des départements universitaires, et une annonce a été publiée sur le site de petites annonces Kijiji. Les participant(e)s avaient tous/toutes le français comme langue maternelle. La majorité (39) était originaire du Québec, 13 venaient de France et les 8 autres d'autres régions de la francophonie (Algérie, Belgique, Gabon, etc.). Trois ont été exclus, dont deux à cause de la vidéo : l'une l'avait déjà visionnée par le passé et l'autre faisait autre chose pendant que le film était diffusé, c'est-à-dire qu'il ne prêtait pas attention. La troisième participante à être exclue avait déjà entendu parler des études de Loftus et disait avoir formulé ses réponses en conséquence, c'est-à-dire qu'elle n'était pas naïve. Le total final est donc de 20 participant(e)s dans la condition de contrôle, 19 dans la condition de présupposition vraie, et 18 dans la condition de présupposition fausse. Chacun(e) recevait une compensation de 20 dollars en argent comptant à la fin de la seconde séance d'expérimentation.

\subsection{Matériel}

\subsubsection{Vidéo}

Après avoir signé leur formulaire de consentement, les participant(e)s ont commencé par regarder la vidéo d'une tentative de vol dans une station-service de Townsville, en Australie, filmée par caméra de surveillance (Queensland Police, 2015). Cette vidéo dure 1 minute 18 secondes. On y voit un homme arrivant dans une camionnette blanche, fracassant la vitre de la porte d'entrée puis entrant à l'intérieur du magasin, une chaîne à la main. La chaîne est constituée d'au moins deux bouts reliés par des crochets; une extrémité est attachée à l'arrière du camion. Tandis que le voleur se dirige vers un coin du magasin qu'on ne distingue pas bien, un des crochets de la chaîne bute contre une dénivellation, dehors, et la chaîne se sépare en deux. Le voleur revient les mains vides, ramasse au passage la masse qu'il a utilisée pour fracasser la vitre de la porte, entre dans son véhicule et démarre, le bout de chaîne encore accroché au camion partant avec lui. Il revient en sens inverse, sort du camion et essaie de relier les deux bouts de chaîne, mais n'y parvient pas parce que le camion est stationné trop loin. Il abandonne alors et repart. La nature comique de la vidéo ainsi que son réalisme nous permettaient à la fois de réduire le risque qu'elle cause des malaises et de nous assurer qu'il s'agissait du type d'événement pour lequel les participant(e)s comprenaient la nécessité d'un témoignage.

Trois questionnaires et une tâche de mémoire de travail ont été soumis aux participant(e)s. 


\subsubsection{Premier questionnaire}

Le questionnaire initial contenant la désinformation existait en trois versions: contrôle, présupposition vraie et présupposition fausse. Pour reproduire ce qui avait déjà été fait (Fiedler et al., 1996; Loftus et al., 1978; Sharman et Powell, 2012), toutes les présuppositions étaient déclenchées par des déterminants définis (le, la). La version de contrôle devait, dans la mesure du possible, ne contenir aucune présupposition. Lorsque c'était possible, les questions critiques dans la version de contrôle ne mentionnaient donc pas du tout l'objet qui était présupposé dans les deux autres versions, c'est-à-dire que si la question contenant une présupposition vraie était «Le voleur a-t-il touché à l'ordinateur sur le comptoir?», la version de contrôle, elle, demandait «Le voleur a-t-il touché à quoi que ce soit sur le comptoir?». Lorsque c'était impossible, la version de contrôle référait à un hyperonyme (vébicule pour pick-up, poubelle pour poubelle jaune) et utilisait un déterminant indéfini (une poubelle au lieu de la poubelle) ou encore elle était précédée par une proposition subordonnée introduite par si («S’il en avait un, de quelle couleur était le véhicule du voleur?»). Dans ce premier exemple (objet sur le comptoir?), il y a quand même une présupposition d'existence d'un objet, mais pas une présupposition du même niveau que celle que nous ciblons. Dans le deuxième cas (usage de déterminants indéfinis), bien que certain(e)s auteur(e)s proposent que des indéfinis puissent être présuppositionnels (voir la discussion de Krifka, 2001), ce n'était pas le cas dans nos exemples. Enfin, dans le dernier cas (utilisation de «si»), la présence d'un antécédent conditionnel qui évoque la possibilité de la non-existence de l'objet présupposé par le conséquent constitue un environnement connu où les présuppositions ne se projettent pas (un «filter» dans le langage de Karttunen, 1973).

Les versions contenant des présuppositions vraies et fausses, au contraire, se concentraient sur des propriétés des objets. Une présupposition vraie mentionnait donc la poubelle jaune, tandis qu'une présupposition fausse mentionnait la poubelle verte; ou alors la présupposition vraie concernait la couleur du pick-up, tandis que la présupposition fausse mettait en doute la couleur du minivan. Les quatre questions critiques sont présentées dans les exemples [12] à [15], où les mots qui changent selon la condition sont présentés en italique pour faciliter la lecture (dans l'ordre: contrôle, présupposition vraie, présupposition fausse). Le questionnaire que les participant(e)s recevaient ne contenait pas d'italique. Tous les mots de vocabulaire dans les questions étaient des mots fréquents, d'usage normal en français (surtout en français québécois); nous avons choisi les mots les plus précis et ordinaires possible, même si cela signifiait utiliser des anglicismes plus familiers (pick-up, par exemple). Nous avons de plus porté attention à certains aspects de référents. Par exemple, il y a à la fois beaucoup de camions (pick-ups) et de fourgonnettes (minivans) au Québec, donc aucun choix n'est a priori plus probable. Nous avons aussi choisi les couleurs de la poubelle en fonction de la réalité de nos participant(e)s (les bacs des poubelles sont bleus, bruns ou noirs au Québec). Évidemment, un des choix pour chaque item était fixé par la réalité de la vidéo, mais nous cherchions toujours un contrepoint avec le même niveau 
de familiarité pour la version contenant la présupposition fausse. Nous avons donc choisi un autre véhicule commun en [12] et une autre couleur inhabituelle en [15] pour éviter un biais au sein d'un seul item. Bien entendu, cela n'empêche pas l'apparition d'effets d'item puisque, justement, certaines questions étaient plus proches de la réalité quotidienne des participant(e)s que d'autres. En effet, nous l'avons évoqué dans la section 2, les participant(e)s ont une meilleure mémoire pour les informations qui correspondent à leur quotidien que pour les informations qui n'y correspondent pas ou qui contrarient même leurs attentes.

[12] Sil en avait un, de quelle couleur était le vébicule/le pick-up/le minivan du voleur?

[13] Le visage du voleur était-il complètement caché?/Le foulard/la cagoule du voleur cachait-il (elle) complètement son visage?

[14] Le voleur a-t-il touché à quoi que ce soit/l'écran d'ordinateur/au téléphone sur le comptoir de la caisse?

[15] Avez-vous vu une poubelle/la poubelle jaune/la poubelle verte? Si oui, où?

Le questionnaire était composé de 15 questions, dont 4 questions critiques. Sur les 15 questions, 5 étaient des questions ouvertes, 8 étaient des questions fermées appelant à une réponse en «oui» ou «non» et 2, enfin, étaient des questions fermées mais appelant à une précision si la réponse était «oui» (par exemple, «Avez-vous vu la plaque d'immatriculation? Si oui, quelle était-elle?»). La distribution complète des questions se trouve dans le tableau 1. Évidemment, un de nos critères était que les informations présupposées (ou non) des stimuli ne pouvaient pas apparaître dans d'autres questions. C'est pourquoi, par exemple, la question sur la plaque d'immatriculation ne nomme pas le type de véhicule. Les études en psychologie ont tendance à se limiter à un seul type de questions - en particulier les questions fermées -, mais, prises ensemble, elles démontrent l'existence d'un effet de désinformation avec chacun de ces types (Dodd et Bradshaw, 1980; Loftus, 1975; Loftus et al., 1978; Sharman et Powell, 2012). Nous avons donc choisi de mélanger les types de questions à des fins de réalisme, puisqu'un enquêteur ne se limite pas à un type de questions. Un effort a été fait pour que les quatre questions critiques soient réparties sur les quatre types de questions; cependant, la question dont la réponse était censée être «oui» («Le visage du voleur était-il complètement caché ?»-alors qu'on voyait ses yeux) s'est révélée vague, de telle sorte qu'un post-test a révélé que la réponse la plus communément admise dans cette situation était en fait «non». En fin de compte, parmi les questions critiques, il y avait donc une question ouverte, deux questions fermées dont la réponse était «non» et une question fermée qui appelait une précision, dont la réponse était «oui». La seizième question du premier questionnaire demandait aux participant(e)s d'écrire en détail ce qu'ils/elles avaient vu dans la vidéo (open recall). La liste complète des questions du sondage, divisée entre les questions critiques et les leurres, se trouve au tableau 3 dans la discussion des résultats. 


\begin{tabular}{|l|l|r|}
\hline Type de questions & Réponse & Nombre \\
\hline Questions ouvertes & & 5 \\
\hline \multirow{2}{*}{ Questions fermées } & Oui & 3 \\
\cline { 2 - 3 } & Non & 5 \\
\hline $\begin{array}{l}\text { Questions fermées } \\
+ \text { précision }\end{array}$ & Oui & 1 \\
\cline { 2 - 3 } Total & Non & 1 \\
\hline
\end{tabular}

Tableau 1 - Distribution des différents types de questions dans le questionnaire initial

\subsubsection{Deuxième questionnaire}

Le deuxième questionnaire était un test de reconnaissance. Il contenait quinze questions à choix multiples dont la première moitié interrogeait des éléments de la vidéo selon leur ordre chronologique d'apparition. La seconde moitié des questions s'intéressait à des éléments de description et était ordonnée du plus général au plus spécifique. Pour les quatre questions critiques (exemples [16]-[19]), réparties logiquement au travers des leurres, les participant(e)s devaient choisir entre l'information véritable issue de la vidéo et l'information fausse (que seul le groupe ayant reçu la version du premier questionnaire contenant des présuppositions fausses avait déjà vue) ${ }^{1}$. Ils/elles devaient ensuite à nouveau fournir un «témoignage écrit» de ce qu'ils/elles avaient vu dans la vidéo.

[16] De quelle couleur était le véhicule? Blanc, noir

[17] Qu'est-ce que le voleur portait sur son visage? Cagoule, foulard

[18] Qu'y avait-il sur le comptoir de la caisse? Téléphone, écran d'ordinateur

[19] De quelle couleur était la poubelle à côté de la porte? Jaune, verte

\subsubsection{Questionnaire démographique}

Le troisième questionnaire recueillait des renseignements sur la situation démographique. Nous avons recueilli l'âge, le genre, l'occupation, la langue maternelle, les langues étrangères parlées et le niveau d'étude.

1. Un(e) expert(e) anonyme a souligné la possibilité très intéressante pour des études ultérieures de présenter trois choix au lieu de deux. Là où nous avons répliqué Loftus et d'autres en donnant un choix fixe entre la bonne réponse et la mauvaise réponse de la condition de présupposition fausse, cet expert aurait inclus une deuxième mauvaise réponse qui n'était pas présente dans la condition de présupposition fausse, l'idée étant qu'il serait plus facile d'identifier l'effet d'une présupposition fausse si les participant(e)s de cette condition choisissent la mauvaise réponse qui correspond à la présupposition fausse plutôt que l'autre réponse incorrecte. 


\subsubsection{Mémoire de travail}

le test de l'empan de lecture de Desmette et al. (1995) a servi de mesure de la mémoire de travail ${ }^{2}$. Les participant(e)s devaient lire à voix haute des blocs de deux à six phrases et rappeler, à la fin de chaque bloc, le dernier mot de chacune des phrases. Les phrases étaient présentées sur ordinateur; l'assistante de recherche faisait apparaitre la prochaine phrase dès que le/la participant(e) avait fini d'en lire une, pour qu'il/elle n'ait pas le temps d'utiliser une stratégie pour mémoriser les mots. Nous avons choisi la version courte pour éviter un effet d'entraînement et pour ne pas allonger exagérément les procédures. Nous avons également préféré, ainsi que le recommandent les auteurs, présenter l'un après l'autre les blocs de phrases d'une longueur croissante plutôt que tous les blocs d'une même longueur à la fois (c'est-à-dire un bloc de deux phrases, un bloc de trois phrases, un bloc de quatre phrases et on recommence, plutôt que les trois blocs de deux phrases, les trois blocs de trois phrases, et les trois blocs de quatre phrases). La proportion des mots cibles rappelée sur le total de 60 mots possibles nous a servi d'indicateur de la capacité de mémoire de travail.

\subsection{Procédure}

Les participant(e)s ont commencé par regarder la vidéo sur un écran d'ordinateur, individuellement. Ils/elles étaient averti(e)s que l'étude portait sur la mémoire et qu'il y aurait un questionnaire sur la vidéo. Cependant, le formulaire de consentement énonçait que la recherche portait sur la qualité visuelle et son influence sur les témoignages subséquents afin de masquer le véritable objectif de la recherche. Nous avons considéré que ceci était un enjeu particulier pour notre étude, car Blank et Launay (2014), entre autres, ont montré qu'un avertissement qu'il pourrait y avoir des erreurs dans les questions du sondage réduit l'influence de la désinformation. Après avoir visionné une seule fois la vidéo, les participant(e)s ont répondu au premier questionnaire, celui qui contenait la désinformation pour un des trois groupes. Les consignes étaient de répondre à toutes les questions du mieux qu'ils/elles le pouvaient; l'assistante de recherche les avertissait qu'elle ne pouvait répondre à aucune question. Elle a néanmoins donné des définitions sommaires à des participant(e)s et, à ceux/celles qui lui ont demandé quoi faire s'ils/si elles ne se souvenaient pas du tout de la réponse, elle a répondu qu'il était possible de donner une réponse au hasard tout en faisant une marque dans la marge.

Un délai variant entre 6 et 15 jours s'est ensuite écoulé avant que les participant(e)s reviennent. La médiane était de 7 jours, et seulement trois personnes ont passé la

2. Ainsi que nos relecteurs ou relectrices nous l'ont fait remarquer, la capacité de mémoire de travail peut varier au cours du temps pour plusieurs facteurs; une mesure de la mémoire de travail lors de la première séance, au moment de la présentation de la vidéo, aurait donc peut-être été plus précise. Notre choix de la placer plutôt à la seconde séance visait la commodité des participant(e)s et la réduction du risque d'influence sur les souvenirs, c'est-à-dire que les participant(e)s auraient pu porter une attention particulière à leurs souvenirs de la vidéo en raison du test de mémoire de travail. 
deuxième partie de l'expérience avec un décalage de plus de 11 jours. Nous avons décidé de garder les résultats de ces participant(e)s étant donné la grande difficulté de recruter des personnes qui se présenteraient plusieurs fois, mais nous avons également ajouté le délai entre les deux séances en tant que facteur à tester dans nos modèles. Au début de la seconde séance d'expérimentation, les participant(e)s recevaient le test de reconnaissance avec les mêmes consignes que pour le premier questionnaire. À la fin du test, l'assistante de recherche leur demandait s'ils/si elles avaient répondu à des questions au hasard, en insistant sur le fait qu'elle ne parlait pas des réponses dont ils/elles n'étaient pas sûr(e)s ou de celles obtenues par déduction. Sans laisser aux participant(e)s l'occasion de changer leurs réponses, elle notait les hasards dans la marge des questionnaires, de même que tous les autres commentaires qu'ils/elles donnaient spontanément.

Les participant(e)s ont ensuite rempli le questionnaire démographique et ont fini avec la tâche de mémoire de travail.

\section{Résultats}

Nous commençons par nos statistiques descriptives avant de présenter les statistiques inférentielles. Le tableau 2 présente le nombre de mauvaises réponses dans le second questionnaire par question selon la condition. Une réponse était comptabilisée comme «bonne» si l'option sélectionnée concordait avec la vidéo; elle était comptabilisée comme «mauvaise» si l'autre option avait été sélectionnée, soit l'option incorrecte par rapport à la vidéo (qui correspondait à l'information faussement présupposée dans une des trois conditions).

Le tableau 3 présente le pourcentage de mauvaises réponses pour toutes les questions au deuxième questionnaire.

La première section du tableau 3 présente les chiffres bruts du tableau 2 en pourcentages. Les deux sections suivantes fournissent les taux de mauvaises réponses aux questions leurres selon que ces questions avaient été posées dans le premier questionnaire ou non, c'est-à-dire que les questions retranscrites dans la deuxième

\begin{tabular}{|l|r|r|r|}
\hline Question & $\begin{array}{l}\text { Présupposition vraie } \\
(n=20)\end{array}$ & Contrôle $(n=19)$ & $\begin{array}{l}\text { Présupposition fausse } \\
(n=18)\end{array}$ \\
\hline Type de véhicule & 0 & 2 & 0 \\
\hline Objet sur le comptoir & 0 & 3 & 8 \\
\hline Vêtement sur le visage & 1 & 3 & 5 \\
\hline Couleur de la poubelle & 3 & 10 & 13 \\
\hline
\end{tabular}

Tableau 2 - Nombre de mauvaises réponses dans le second questionnaire par question critique selon la condition 


\begin{tabular}{|c|c|c|c|}
\hline Question & $\begin{array}{l}\text { Présupposition } \\
\text { vraie }(n=20)\end{array}$ & Contrôle $(n=19)$ & $\begin{array}{l}\text { Présupposition } \\
\text { fausse }(n=18)\end{array}$ \\
\hline \multicolumn{4}{|l|}{ Questions critiques } \\
\hline $\begin{array}{l}\text { Quel type de véhicule le voleur } \\
\text { avait-il? }\end{array}$ & 0,00 & 0,11 & 0,00 \\
\hline $\begin{array}{l}\text { Qu'y avait-il sur le comptoir de } \\
\text { la caisse? }\end{array}$ & 0,00 & 0,16 & 0,44 \\
\hline $\begin{array}{l}\text { Qu'est-ce que le voleur portait } \\
\text { sur son visage? }\end{array}$ & 0,05 & 0,16 & 0,28 \\
\hline $\begin{array}{l}\text { De quelle couleur était } \\
\text { la poubelle à côté de la porte? }\end{array}$ & 0,15 & 0,53 & 0,72 \\
\hline \multicolumn{4}{|l|}{ Questions incluses dans le Q. 1} \\
\hline Était-ce la nuit ou le jour? & 0,00 & 0,05 & 0,00 \\
\hline $\begin{array}{l}\text { Qu'est-ce que le voleur essayait } \\
\text { de voler? }\end{array}$ & 0,05 & 0,05 & 0,11 \\
\hline $\begin{array}{l}\text { De quelle couleur était } \\
\text { le véhicule? }\end{array}$ & 0,05 & 0,11 & 0,17 \\
\hline $\begin{array}{l}\text { Qu'est-ce que le voleur a d'abord } \\
\text { lancé dans la vitre? }\end{array}$ & 0,20 & 0,37 & 0,22 \\
\hline $\begin{array}{l}\text { De quelle couleur était la veste } \\
\text { du voleur? }\end{array}$ & 0,35 & 0,32 & 0,44 \\
\hline \multicolumn{4}{|l|}{ Questions non incluses dans le Q. 1} \\
\hline $\begin{array}{l}\text { Quelle vitre le voleur a-t-il } \\
\text { brisée? }\end{array}$ & 0,05 & 0,05 & 0,00 \\
\hline $\begin{array}{l}\text { Qu'est-ce que le voleur a utilisé } \\
\text { pour finir de fracasser la vitre? }\end{array}$ & 0,05 & 0,16 & 0,11 \\
\hline $\begin{array}{l}\text { Quel instrument y avait-il dans la } \\
\text { remorque du voleur? }\end{array}$ & 0,35 & 0,11 & 0,11 \\
\hline $\begin{array}{l}\text { Qu'est-ce que le présentoir que la } \\
\text { chaîne a accroché contenait? }\end{array}$ & 0,35 & 0,32 & 0,28 \\
\hline $\begin{array}{l}\text { Qu'est-ce que le voleur portait } \\
\text { sur les mains? }\end{array}$ & 0,55 & 0,53 & 0,33 \\
\hline $\begin{array}{l}\text { De quel côté du véhicule le } \\
\text { voleur entrait et sortait-il? }\end{array}$ & 0,40 & 0,68 & 0,83 \\
\hline
\end{tabular}

Tableau 3 - Taux de mauvaises réponses dans le second questionnaire par question selon la condition, pour toutes les questions 
section du tableau avaient été posées dans le premier questionnaire tandis que les questions de la troisième section du tableau étaient nouvelles. Le premier questionnaire contenait 15 questions, dont 6 n'ont pas été posées de nouveau dans le questionnaire final. Les questions ont été réorganisées dans le tableau pour faciliter la comparaison des données et leur classement ne correspond donc pas à l'ordre dans lequel les participant(e)s les recevaient. Les questions leurres ont été exclues des analyses statistiques puisqu'elles étaient identiques pour toutes les conditions et ne cadraient donc pas dans notre objet de recherche.

Nous avons également modélisé les réponses aux questions critiques à l'aide de régressions logistiques binomiales à effets aléatoires croisés; la variable indépendante était la condition, la variable dépendante, la nature correcte ou incorrecte des réponses au deuxième questionnaire, et les effets aléatoires, les participant(e)s et les questions. L'analyse a été faite dans le logiciel statistique R (version 3.3.3) (R Core Team, 2000) à l'aide de la fonction «glmer» de la bibliothèque «lme4» (Bates et al., 2014). Le tableau 4 présente donc l'influence de la présupposition vraie, de la présupposition fausse, du délai entre les deux questionnaires et de la mémoire de travail sur la probabilité de fournir une bonne réponse aux questions critiques telle que calculée pour la condition contrôle.

Dans la colonne «p» du tableau, le modèle révèle que l'exposition à une présupposition vraie augmentait effectivement les probabilités d'obtenir une bonne réponse au deuxième questionnaire, étant donné que $p<0,01$, tandis que l'exposition à une présupposition fausse s'approche d'être un facteur significatif sans tout à fait l'atteindre $(p=0,087)$. La longueur des délais entre les deux séances d'expérimentation, lorsqu'ajoutée au modèle, n'atteignait pas la significativité $(p=0,125)$; de même, la régression logistique calculée sur les données excluant les deux participantes dont le délai entre les deux séances était supérieur à onze jours ne présentait pas de différence notable.

\begin{tabular}{|l|r|r|r|r|r|r|r|}
\hline & Estimé & $\begin{array}{l}\text { Erreur- } \\
\text { type }\end{array}$ & Valeur $Z$ & $p$ & O. R. & \multicolumn{2}{|l|}{ Intervalle de confiance } \\
\cline { 6 - 8 } & 1,978 & 0,637 & 3,103 & $p=0,002$ & 7,229 & 2,072 & 25,215 \\
\hline $\begin{array}{l}\text { Présupposition } \\
\text { vraie }\end{array}$ & $-0,721$ & 0,421 & $-1,712$ & $p=0,087$ & 0,486 & 0,213 & 1,110 \\
\hline $\begin{array}{l}\text { Présupposition } \\
\text { fausse }\end{array}$ & 0,217 & 0,142 & 1,533 & $p=0,125$ & 1,243 & 0,941 & 1,640 \\
\hline Délai & $-1,407$ & 2,013 & $-0,699$ & $p=0,485$ & 0,245 & 0,005 & 12,653 \\
\hline $\begin{array}{l}\text { Mémoire de } \\
\text { travail }\end{array}$ & & & & & & &
\end{tabular}

Tableau 4 - Analyse inférentielle ${ }^{3}$

3. L'influence de la mémoire de travail a été analysée à l'aide d'un modèle séparé sur un échantillon plus petit. 


\section{Discussion}

Du côté de la mémoire de travail, une analyse de variance (ANOVA) a d'abord été effectuée sur les valeurs obtenues pour vérifier que le fait que le test ait été construit en français hexagonal n'avantageait pas les participant(e)s français(es). Les participant(e)s ont été séparé(e)s en trois groupes selon leur origine, soit Québec, France et autres. La mémoire de travail des trois groupes formait une courbe normale, ce qui justifiait l'utilisation de l'ANOVA. Celle-ci n'a pas détecté d'avantage dialectal, $\mathrm{F}(2,54)=2,35$, $p=0,105$, ce qui nous a permis de conduire l'analyse. Cette dernière a été effectuée sur un modèle séparé, construit sur les données de 54 participant(e)s, au contraire de l'autre modèle, qui prenait en compte 57 participant(e)s. Il nous semblait en effet judicieux de ne pas exclure du modèle principal les participant(e)s dont la mesure de mémoire de travail n'était pas recevable, mais dont le reste des données l'était. Ce second modèle révèle que la mémoire de travail, dans cette expérience, n'a pas d'effet significatif sur l'obtention de bonnes réponses au second questionnaire. L'indice de mémoire de travail moyen que nous avons obtenu est de 29,48 \% (écart-type: $11,38 \%$ ) de mots rappelés, comparé à la moyenne normale de 34,75\% (écart-type : 13,4 \%) de mots rappelés (Desmette et al., 1995).

Par la suite, les résultats de chacune des quatre questions critiques ont été individuellement soumis à une régression logistique avec le/la participant(e) pour seul effet aléatoire. À cause des cellules vides, l'analyse n'est pas concluante pour la question du véhicule ni pour la question de l'objet sur le comptoir. En ce qui concerne la question de la poubelle, la différence de bonnes réponses dans les conditions de contrôle et de présupposition vraie est significative $(p<0,05)$, mais pas celle entre les conditions de contrôle et de présupposition fausse. Finalement, il n'y a pas de différence statistiquement significative entre les conditions pour la question sur le vêtement qui couvre le visage du voleur. Une autre régression logistique avec les participant(e)s comme effet aléatoire et les questions comme facteur testé confirme l'influence importante de chacune des questions dans le modèle $(p<0,01)$.

Rappelons maintenant que l'étude de Loftus (1975) a montré une différence significative entre une condition de présupposition fausse et une condition de contrôle, et, dans une expérience différente, une différence significative entre une condition de présupposition vraie et une condition de contrôle, et enfin que l'étude de Loftus et al. (1978) a indiqué une divergence entre ces mêmes conditions quand elles étaient testées ensemble, bien qu'il n'y ait pas eu assez de participant(e)s pour discuter de la significativité de ce résultat. Nos résultats sont ici intéressants: la distinction entre la condition de présupposition vraie et la condition de contrôle est significative, mais la distinction originellement observée dans la première étude entre la condition de présupposition fausse et la condition de contrôle ne l'est pas.

Le but premier de cette étude était d'abord de vérifier si l'effet de désinformation trouvé en anglais par Loftus et ses successeurs est généralisable à d'autres langues, ici le français. Notre expérience pose autant de questions qu'elle apporte de réponses à 
ce sujet. Si nous n'avons pas trouvé d'effet de désinformation significatif, la tendance des présuppositions fausses à éliciter des mauvaises réponses était juste assez forte pour soulever la question de la puissance statistique. Ainsi, il est possible que nos résultats s'approcheraient plus de ceux de Loftus (1975) et de Loftus et al. (1978) si nous avions eu un échantillon plus important, ce qui serait cohérent avec les études qui ont pu reproduire l'effet en allemand ou en chinois (Wyler et Oswald, 2016; Zhu et al., 2010). Cependant, il ne faudrait pas rejeter d'emblée l'hypothèse que cet écart soit dû, au moins en partie, à une différence culturelle ou linguistique. En effet, d'autres études ont réussi à obtenir un effet de désinformation significatif avec des échantillons semblables au nôtre (Fiedler et al., 1996), ou même plus petits (Jaschinski et Wentura, 2002).

De l'autre côté, la différence entre le taux de bonnes réponses des participants exposés à des présuppositions vraies et celui des participant(e)s en condition de contrôle est significative. Un(e) participant(e) exposé(e) à des présuppositions vraies aurait entre 2 et 25 fois plus de chances de donner la bonne réponse à un test ultérieur. Considérant que ces chiffres ont été obtenus dans les mêmes conditions d'expérimentation que ceux de la présupposition fausse, cela semble indiquer que l'effet de consolidation des présuppositions vraies sur la mémoire est plus puissant et potentiellement plus fiable que l'effet de désinformation. Une tendance similaire peut être observée parmi les données de Loftus (1975, exp. 1), où les personnes ayant été exposées à des présuppositions vraies ont un taux de bonnes réponses de $75 \%$, comparé à $41 \%$ pour les personnes exposées à des présuppositions fausses. En supposant qu'une personne répondant au hasard obtiendrait un taux de $50 \%$, on obtient un écart présupposition vraie-hasard de $25 \%$ qui est plus large que l'écart présupposition fausse-hasard, de $9 \%$. En revanche, dans l'étude de Loftus et al. (1978), qui introduit un groupe de contrôle, sûrement un meilleur indicateur que le hasard, l'effet est inversé : l'effet positif d'une présupposition vraie est de $7 \%$ seulement, tandis que l'effet négatif d'une présupposition fausse est presque trois fois plus fort $(20 \%)$. Le tableau 5 compare les pourcentages pour leur étude et notre reproduction de la même expérience.

Malgré les tendances similaires dans l'ordre des différents groupes quand ils sont classés par proportion de faux rapports, nous constatons des différences assez marquées. D’abord, pour notre échantillon, même avec une présupposition fausse, à peine plus d'un tiers des participant(e)s ont donné une mauvaise réponse, ce qui

\begin{tabular}{|l|r|r|}
\hline & Loftus et al., 1978 & La présente étude \\
\hline Présupposition vraie & $30 \%$ & $5 \%$ \\
\hline Contrôle & $36,67 \%$ & $23,68 \%$ \\
\hline Présupposition fausse & $56,67 \%$ & $36,11 \%$ \\
\hline
\end{tabular}

Tableau 5 - Proportion de faux rapports selon la condition au travers de deux études 
est déjà plus bas que le groupe de contrôle de Loftus et al. (1978). D'ailleurs, là où nos participant(e)s ont reçu des questions à présupposition vraie, l'erreur possible pour un témoin oculaire est réduite à $5 \%$, ce qui est d'autant plus incroyable quand on voit que certaines questions ont porté sur des objets qui étaient extrêmement périphériques (comme la poubelle), qui sont pourtant plus sujets à la désinformation que les objets centraux (Wyler et Oswald, 2016). Notre modèle statistique confirme la significativité de cette tendance : la présupposition vraie a réduit le taux d'erreur de plus de $18 \%$ tandis que la présupposition fausse l'a haussé de moins de $13 \%$. Même si nous ne pouvons pas comparer nos résultats de façon formelle, nous trouvons intéressante la possibilité que la population testée par notre étude subisse moins l'influence négative des présuppositions fausses, mais ressente plus l'influence positive des présuppositions vraies que l'échantillon testé par Loftus et ses collègues.

Certaines réserves doivent être émises vis-à-vis de ces résultats qui peuvent sembler provocateurs. D'abord, ce n'est pas parce qu'un effet n'est pas significatif qu'on peut conclure qu'il n'y a pas d'effet; il est donc tout à fait possible que des études ultérieures montrent un manque d'effet langagier ou culturel par rapport à l'effet des questions désinformantes. Ensuite, nous avons reconstitué la conception de l'étude de Loftus et ses collègues assez fidèlement, et ce faisant, nous avons reproduit ses forces ainsi que ses faiblesses. En particulier, Loftus et al. ont choisi un modèle intergroupe alors qu'il serait plus approprié d'utiliser un schéma intragroupe de carré latin. C'est important pour le groupe qui a répondu à quatre questions avec des présuppositions fausses. Il se peut en effet qu'un(e) participant(e) ait été alerté(e) par une de ces questions et qu'il/elle ait été plus vigilant(e) envers les autres par la suite. Dans ce cas, nous nous attendrions à ce que l'effet de la condition de présupposition fausse soit diminué, et étant donné le manque de significativité de la différence entre cette condition et la condition de contrôle, il est possible que ce soit effectivement le cas ici. Si les questions relevant du contrôle, de la présupposition fausse et de la présupposition vraie avaient été équilibrées, cela aurait permis de compenser un tel effet. Cela étant dit, nous avons tenté de minimiser les risques d'apparition d'une telle vigilance, notamment en incluant beaucoup de leurres qui ne contenaient que des présuppositions vraies. D'ailleurs, l'ordre dans lequel les items étaient présentés est peut-être intéressant. La question sur le véhicule était celle qui induisait le moins de personnes en erreur (zéro, en fait), et elle était la première à être posée parmi les questions critiques. La question suivante (à savoir une présupposition fausse) était celle de l'objet sur le comptoir et, comme le tableau 2 le montre, elle a induit beaucoup de participant(e)s en erreur (avec un écart plus grand pour cette question entre contrôle et présupposition fausse qu'entre contrôle et présupposition vraie). Nous pensions que des participant(e)s pourraient corriger des questions incorrectes, mais cela n'est arrivé qu'une seule fois avec une seule question (celle du véhicule). Néanmoins, une étude complémentaire suivant le modèle du carré latin sera un ajout important à l'avenir.

Afin de répondre à ces deux problèmes possibles, nous avons déjà créé une version de notre expérience avec six questions critiques, y compris les quatre questions originales, dans un modèle en carré latin, sur Amazon Mechanical Turk, un site de 
recrutement de travailleurs et de travailleuses à travers le monde entier qui peuvent participer à des tâches variées. Malgré le défi de s'assurer que les participant(e)s à l'extérieur du laboratoire portent attention aux vidéos et qu'ils/elles répondent immédiatement aux questions, etc., si nous réussissons à reproduire nos propres résultats pour la même vidéo et les mêmes questions (ainsi que deux questions supplémentaires), nous comptons faire passer la même expérience (traduite en anglais) aux anglophones au Québec ainsi qu'aux anglophones aux États-Unis. Cela nous permettra de mieux trancher à la fois entre un effet de culture et de langue en comparant, d'un côté, des francophones et des anglophones habitant dans la même province et, de l'autre, des anglophones de deux pays différents. L'avantage de cibler les Américain(e)s serait aussi de comparer les résultats à ceux obtenus par Loftus plus directement, mais avec des stimuli différents et 40 ans plus tard.

Enfin, contrairement à Jaschinski et Wentura (2002), nous n'avons pas trouvé d'effet significatif de la mémoire de travail sur le taux de bonnes réponses. Le test statistique utilisé y est peut-être pour quelque chose: Jaschinski et Wentura (2002), ayant testé la désinformation pour chacun(e) des participant(e)s, ont pu fabriquer un «indice de désinformation» à partir de l'écart individuel entre le nombre de bonnes réponses en condition de contrôle et le nombre de bonnes réponses en condition de désinformation. Ils ont donc pu corréler leur indice de désinformation avec l'indice de mémoire de travail. En ce qui nous concerne, nous avons plutôt monté un modèle statistique et vérifié à quel point la mémoire de travail pouvait expliquer les modulations de bonnes réponses par rapport aux autres facteurs comme la condition. Malgré tout, il est assez étonnant de n'avoir pas reproduit un effet de corrélation entre la mémoire de travail et le taux de fausses réponses de la perspective de la littérature en psychologie. Nous avons donc regardé d'autres possibilités de près. Nous avons confirmé qu'il y avait de la variabilité dans les résultats du test de mémoire de travail et que ceux-ci étaient distribués selon une courbe normale. Nous avons vérifié que tout ce qui avait été contrôlé pour la version en anglais du test de mémoire de travail avait été préservé dans les contrôles de sa traduction en français (fréquence des mots, catégorie syntaxique du dernier mot, etc.). Il est également possible que notre population étudiante, surtout considérant l'ajout des membres de la communauté recrutés via le site de petites annonces, soit différente de la population recrutée pour les études de Loftus et qu'une telle différence produise cet effet. Il y a au moins une autre étude qui a cherché à établir une corrélation entre la désinformation et la mémoire de travail sans la trouver: celle de Corley (2015). Il est vrai que, d'emblée, la mémoire de travail n'est pas aussi pertinente pour nos souvenirs que notre mémoire à long terme, par exemple, donc nous n'excluons pas la possibilité que ce résultat reflète quelque chose de plus profond.

Revenant finalement aux notions théoriques de la présupposition, nous espérons que cette étude pourra constituer une base pour d'autres recherches plus approfondies dans le domaine linguistique, en commençant par l'évaluation de l'effet de différentes classes de présuppositions. Nous trouvons également intéressante l'idée qu'une étude semblable, où les participant(e)s porteraient cette fois un dispositif de suivi des yeux, 
pourrait donner de meilleurs résultats par rapport à ce qui fait véritablement partie du savoir partagé, puisque l'attention visuelle du/de la participant(e) s'attarderait là-dessus. Nous pourrions commencer à tenter de distinguer des effets d'item en démêlant l'appropriété et la vérité des présuppositions, ce qui mènerait naturellement à une meilleure compréhension des facteurs contribuant à l'accommodation des présuppositions ainsi que de leur effet sur la perméabilité de la mémoire d'événements à long terme.

\section{Conclusion}

En conclusion, il n'est pas certain que l'effet de désinformation soit un phénomène universel applicable à toutes les cultures et à toutes les langues de la même façon. Sans dire qu'une langue ou une culture pourrait être complètement immunisée contre cet effet, nous soulevons l'idée que certains mécanismes pragmatiques et certaines valeurs culturelles puissent affecter la puissance de l'effet de désinformation. Il a ainsi déjà été démontré que l'assertion, une méthode de partage de l'information moins insidieuse et moins contraignante pour l'interlocuteur ou l'interlocutrice, suscite un effet de désinformation moins puissant que la présupposition (Fiedler et al., 1996); si l'effet de désinformation varie à l'intérieur même d'une langue, il semble probable qu'il varie également d'une langue à l'autre, sous l'influence de mécanismes linguistiques et pragmatiques différents. De plus, il nous apparaît plausible qu'une culture plus critique vis-à-vis de l'information et de sa source soit moins affectée qu'une culture où il est socialement inacceptable de reprendre son interlocuteur ou interlocutrice. Il serait à cet égard intéressant de tester l'effet de désinformation sur des langues dans lesquelles les marqueurs d'évidentialité sont obligatoires.

Par ailleurs, le constat le plus intéressant de cette étude est que l'effet de consolidation de la présupposition vraie est plus important que l'effet de désinformation de la présupposition fausse. Cela nous semble particulièrement important pour le domaine judiciaire: le simple fait de répéter, sous forme de présupposition, une information déjà constatée par un témoin, pourrait renforcer son souvenir de cette information et en enrayer la dégradation. D'autres études pourront vérifier si l'exposition à des présuppositions vraies augmente la résistance à la désinformation.

\section{Références}

Baddeley, A. 2003. Working Memory: Looking back and Looking forward. Nature Reviews Neuroscience 4 (10): 829-839.

Bates, D., Maechler, M., Bolker, B. et Walker, S. 2014. «lme4»: Linear Mixed-Effects Models Using «Eigen» and S4. En ligne à l'adresse suivante: https://cran.r-project. org/package $=1 \mathrm{me} 4$.

Beaver, D. I. 2001. Presupposition and Assertion in Dynamic Semantics. Stanford: CSLI Publications. 
Beaver, D. I. et Geurts, B. 2011. Presupposition. Stanford Encyclopedia of Philosophy. En ligne à l'adresse suivante: https://plato.stanford.edu/entries/presupposition/.

Blank, H. et Launay, C. 2014. How to Protect Eyewitness Memory against the Misinformation Effect: A Meta-Analysis of Post-Warning Studies. Journal of Applied Research in Memory and Cognition 3 (2): 77-88.

Chrobak, Q. M. et Zaragoza, M. S. 2013. The Misinformation Effect: Past Research and Recent Advances. In A. M. Ridley, F. Gabbert et D. J. La Rooy (éd.), Suggestibility in Legal Contexts: Psychological Research and Forensic Implications. Malden - Oxford: Wiley-Blackwell: 21-44.

Corley, W. B. 2015. The Effect of Forewarning on Suggestibility: Does It Depend on WorkingMemory Capacity? Mémoire de master. Western Kentucky University. En ligne à l'adresse suivante: http://digitalcommons.wku.edu/theses/1497/.

Davis, J. et Schiffman, H. R. 1985. The Influence of the Wording of Interrogatives on the Accuracy of Eyewitness Recollections. Bulletin of the Psychonomic Society 23 (4) : 394-396.

Desmette, D., Hupet, M., Schelstraete, M.-A. et Van Der Linden, M. 1995. Adaptation en langue française du «Reading Span Test» de Daneman et Carpenter (1980). L'Année psychologique 95 (3): 459-482.

Dodd, D. H. et Bradshaw, J. M. 1980. Leading Questions and Memory: Pragmatic Constraints. Journal of Verbal Learning and Verbal Bebavior 19 (6) : 695-704.

Ducrot, O. 1968. La description sémantique des énoncés français et la notion de présupposition. L'Homme 8 (1): 37-53.

Ducrot, O. 1969. Présupposés et sous-entendus. Langue française 4: 30-43.

Fiedler, K., Walther, E., Armbruster, T., Fay, D. et Naumann, U. 1996. Do You Really Know What You Have Seen? Intrusion Errors and Presuppositions Effects on Constructive Memory. Journal of Experimental Social Psychology 32 (5) : 484-511.

Fintel, K. von 2008. What Is Presupposition Accommodation, Again? Philosophical Perspectives 22 (1): 137-170.

Frege, G. 1956. The Thought: A Logical Inquiry. Mind 65 (259) : 289-311.

Jaschinski, U. et WenturA, D. 2002. Misleading Postevent Information and Working Memory Capacity: An Individual Differences Approach to Eyewitness Memory. Applied Cognitive Psychology 16 (2) : 223-231.

Karttunen, L. 1973. Presuppositions of Compound Sentences. Linguistic Inquiry 4 (2): 169-193.

Karttunen, L. 1974. Presupposition and Linguistic Context. Theoretical Linguistics 1 (1-3): 181-194.

Karttunen, L. 2016. Presupposition: What Went Wrong? In M. Moroney, C.-R. Little, J. Collard et D. Burgdorf (éd.), Proceedings of the 26th Semantics and Linguistic Theory Conference (Austin, University of Texas, 12-15 May 2016). Washington : Linguistic Society of America: 705-731. En ligne à l'adresse suivante: https://journals.linguisticsociety. org/proceedings/index.php/SALT/article/download/26.705/3668.

KrIFKA, M. 2001. Non-Novel Indefinites in Adverbial Quantification. In C. A. Condoravdi et G. Renardel de Lavalette (éd.), Logical Perspectives on Language and Information. Stanford: CSLI Publications: 1-40. 
Liebman, J. I., McKinley-Pace, M. J., Leonard, A. M., Sheesley, L. A., Gallant, C. L., Renkey, M. E. et Lehman, E. B. 2002. Cognitive and Psychosocial Correlates of Adults' Eyewitness Accuracy and Suggestibility. Personality and Individual Differences 33 (1): 49-66.

Loftus, E. F. 1975. Leading Questions and the Eyewitness Report. Cognitive Psychology $7(4): 560-572$.

Loftus, E. F. 1977. Shifting Human Color Memory. Memory and Cognition 5 (6) : 696-699.

Loftus, E. F., Miller, D. G. et Burns, H. J. 1978. Semantic Integration of Verbal Information into a Visual Memory. Journal of Experimental Psychology: Human Learning and Memory 4 (1): 19-31.

McCloskey, M. et Zaragoza, M. S. 1985. Misleading Postevent Information and Memory for Events: Arguments and Evidence against Memory Impairment Hypotheses. Journal of Experimental Psychology: General 114 (1): 1-16.

QueEnsland Police 2015. Attempted ATM Theft, Townsville. En ligne à l'adresse suivante : https://www.youtube.com/watch?v=7hD6nNcvBu0.

Russell, B. 1905. On Denoting. Mind 14 (56) : 479-493.

Schmiedek, F., LövdÉn, M. et Lindenberger, U. 2014. A Task Is a Task Is a Task: Putting Complex Span, $N$-Back, and Other Working Memory Indicators in Psychometric Context. Frontiers in Psychology 5 (1475): 1-8.

Sharman, S. J. et Powell, M. B. 2012. A Comparison of Adult Witnesses' Suggestibility across Various Types of Leading Questions. Applied Cognitive Psychology 26 (1) : 48-53.

Stalnaker, R. 1973. Presuppositions. Journal of Philosophical Logic 2 (4) : 447-457.

Stalnaker, R. 1974. Pragmatic Presuppositions. In M. K. Munitz et P. K. Unger (éd.), Semantics and Philosopby. New York: New York University Press: 197-213.

Strawson, P. F. 1950. On Referring. Mind 59 (235) : 320-344.

R Core Team 2000. R Language Definition. Vienne: R Foundation for Statistical Computing. En ligne à l'adresse suivante: https://cran.r-project.org/doc/manuals/R-lang.html.

VAN Der SAndt, R. A. 2015. Linguistic Presupposition. In N. J. Smelser et P. B. Baltes (éd.), International Encyclopedia of the Social and Behavioral Sciences. Amsterdam Paris - New York: Elsevier : 190-192.

Vornik, L., Sharman, S. et Garry, M. 2003. The Power of the Spoken Word: Sociolinguistic Cues Influence the Misinformation Effect. Memory 11 (1): 101-109.

Wyler, H. et Oswald, M. E. 2016. Why Misinformation Is Reported: Evidence from a Warning and a Source-Monitoring Task. Memory 24 (10) : 1419-1434.

Zaragoza, M. S., Belli, R. F. et Payment, K. E. 2007. Misinformation Effects and the Suggestibility of Eyewitness Memory. In M. Garry et H. HAYne (éd.), Do Justice and Let the Sky Fall: Elizabeth F. Loftus and Her Contributions to Science, Law, and Academic Freedom. Mahwah: L. Erlbaum: 35-63.

Zhu, B., Chen, C., Loftus, E. F., Lin, C., He, Q., Chen, C., Li, H., Xue, G., Lu, Z. et Dong, Q. 2010. Individual Differences in False Memory from Misinformation: Cognitive Factors. Memory 18 (5): 543-555. 